\title{
Institucionalidad Antártica Chilena ${ }^{1}$
}

\section{Chilean Antarctic Institutionality}

\section{Camilo Sanhueza Bezanilla}

csanhueza@minrel.gob.cl

Ministro Consejo. Director de Antártica, Ministerio de Relaciones Exteriores de Chile.

Resumen: Se analizan las instituciones con competencias antárticas dentro de Estado de Chile así como los documentos directivos de la actividad antártica nacional. Se revisan las reuniones y foros internacionales en materia antártica que Chile participa. Finalmente se hacen algunos alcances respecto a la futura Ley Antártica Chilena. Todo lo anterior permite tener una visión general sobre la institucionalidad antártica chilena.

Palabras claves: Instituciones chilenas con competencias antárticas - Sistema del Tratado Antártico - Actividad antártica chilena - Ley Antártica Chilena

\begin{abstract}
The Chilean institutions with competence in Antarctic issues and the directive documents about it are analyzed. The international Antarctic meetings and forums where Chile participates are also reviewed. Finally, the main aspects of the new Chilean Antarctic Law are highlighted. All this explanation gives an overview of the Chilean Antarctic institutionality.
\end{abstract}

Keywords: Chilean Institutions with competence in Antarctic issues - Antarctic Treaty System Chilean Antarctic Activities - Chilean Antarctic Law

\section{Introducción}

Chile tiene una larga tradición antártica, fundada en razones geográficas, históricas y jurídicas. En este sentido nuestra Política Antártica ha sido definida sobre la base de esos elementos y contempla dos componentes, por una parte en el ámbito internacional impulsando la activa

1 Ponencia presentada en las Jornadas Chilenas de Derecho Antártico 2019, Facultad de Derecho, Universidad de Chile, 28 de marzo del 2019.

Número de página no utilizable para citar

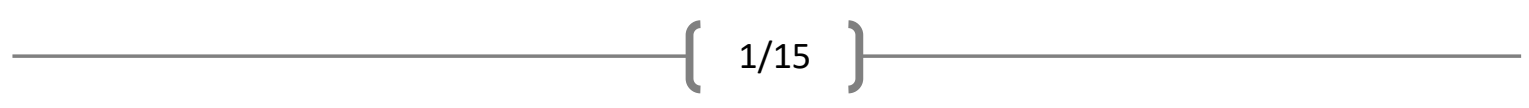


participación en la gestación, implementación y desarrollo de un régimen antártico efectivo a partir del Tratado Antártico y, por la otra, un componente de derecho interno esencial para dar eficacia a los principios y normas internacionales, así como para el ejercicio de las competencias del Estado chileno en el continente, y particularmente en el Territorio Chileno Antártico.

Esta dualidad de la Política Antártica chilena obliga a actuar responsablemente tanto en el seno del Tratado Antártico como en el ámbito interno, buscando siempre ejercer de forma integral los derechos que corresponden a Chile.

\section{Instituciones y directivas nacionales}

\subsection{Ministerio de Relaciones Exteriores}

\subsubsection{Generalidades}

Al Ministerio de Relaciones Exteriores, le corresponde el conocimiento y coordinación de todos los asuntos relativos al Territorio Chileno Antártico y a la Antártica en general, velando por que las actividades que se desarrollen en dicho continente se ajusten a los lineamientos de la Política Antártica Nacional y sean acordes con las normas internacionales que obligan a Chile.

Asimismo, le corresponde colaborar con el Presidente de la República en la planificación, dirección, coordinación, ejecución, control e información de la política exterior de Chile en relación al Continente Antártico, y asumir la representación nacional ante las instancias del Sistema del Tratado Antártico y las relaciones bilaterales sobre la materia.

Los diversos ministerios, tales como el de Defensa Nacional, Medio Ambiente y Economía, entre otros, así como entidades estatales con competencia sectorial en las actividades antárticas, coordinarán su labor por medio del Ministerio de Relaciones Exteriores.

Son funciones del Ministerio de Relaciones Exteriores el supervisar y coordinar la ejecución de la Política Antártica Nacional; la coordinación de la realización de las actividades nacionales en la Antártica en el marco del Programa Antártico Nacional; el asesorar al Presidente de la República en la conducción de los aspectos políticos y diplomáticos de la Política Antártica; mantener las relaciones multilaterales y bilaterales con los Estados, entidades internacionales, instancias y regímenes internacionales del Sistema del Tratado Antártico, entre otras.

Número de página no utilizable para citar 
Asimismo, el Ministerio de Relaciones Exteriores tendrá la función de desarrollar los Planes Estratégicos Antárticos, en base a los objetivos que establezca la Política Antártica Nacional, los cuales comprenderán las tareas y acciones concretas que se materializarán mediante el Programa Antártico Nacional de cada año.

En consecuencia, para el desarrollo de su tarea en el ámbito antártico, el Ministerio debe relacionarse y coordinarse tanto en el ámbito nacional como internacional.

\subsubsection{El Consejo de Política Antártica}

El Consejo de Política Antártica es el órgano colegiado de naturaleza interministerial, presidido por el Ministro de Relaciones Exteriores, que tiene por función proponer al Presidente de la República las bases políticas, jurídicas, científicas, económicas, medioambientales, logísticas, deportivas, culturales, y de difusión de la acción nacional en la Antártica y proponer los grandes lineamientos de la Política Antártica Nacional.

Respecto a sus funciones e integración se regirá por lo dispuesto en el Estatuto Orgánico del Ministerio de Relaciones Exteriores, Ley 21.080 del 2018, la cual modifica diversos cuerpos legales con el objeto de modernizar el Ministerio de Relaciones Exteriores, y por un nuevo reglamento que deberá dictarse dentro de los próximos meses.

El Consejo de Política Antártica estará integrado por el Ministro de Relaciones Exteriores o quien lo subrogue, quien lo presidirá; el Ministro de Defensa Nacional; el Ministro de Hacienda; el Ministro de Economía, Fomento y Turismo; el Ministro del Medio Ambiente y el Jefe del Estado Mayor Conjunto.

Además, participarán como asesores, el Subsecretario de Relaciones Exteriores; el Subsecretario de Defensa; el Subsecretario de Pesca y Acuicultura; el Comandante en Jefe del Ejército de Chile; el Comandante en Jefe de la Armada de Chile; el Comandante en Jefe de la Fuerza Aérea de Chile; el Delegado Presidencial de la Región de Magallanes y Antártica Chilena; el Director Nacional de Fronteras y Límites del Estado; el Director del Instituto Antártico Chileno; el Director de Planificación Estratégica de la Subsecretaría de Relaciones Exteriores y el Director de Antártica del Ministerio de Relaciones Exteriores o el funcionario de tal cartera encargado de los temas antárticos, quién actuará, además, como Secretario Ejecutivo del Consejo.

El Ministro de Relaciones Exteriores podrá invitar a representantes del Congreso Nacional, ministerios, órganos de la Administración del Estado, operadores antárticos, y representantes de la sociedad civil, para los efectos de realizar consultas sobre materias específicas.

Número de página no utilizable para citar 


\subsubsection{Dirección de Antártica}

La Dirección de Antártica (DIRANTARTICA) es la responsable de centralizar los asuntos antárticos y planificar la conducción y ejecución de sus aspectos diplomáticos, jurídicos y políticos en conjunto con los organismos nacionales e internacionales pertinentes, cautelando el interés nacional en la conservación y protección de la Antártica y los derechos soberanos de Chile sobre el Territorio Chileno Antártico, sector que se proyecta hasta el Polo Sur, entre los meridianos $53^{\circ}$ y $90^{\circ}$ de longitud oeste de Greenwich.

Esta Dirección concentra todos los aspectos correspondientes al funcionamiento del Sistema del Tratado Antártico y las relaciones con sus Estados Partes, cumpliendo cabalmente las obligaciones internacionales originadas por este sistema, sus instrumentos y mecanismos consultivos en la administración del Continente Antártico.

Coordina la aplicación de la Política Antártica Nacional, del Plan Estratégico Antártico y colabora junto a otras entidades públicas competentes en el posicionamiento de Chile como País Puente, y de la Región de Magallanes y Antártica Chilena como polo de servicios y vía preferente de acceso hacia la Antártica sudamericana, velando por el desarrollo de las actividades productivas en el Continente Antártico con estricto apego a las normas de este régimen internacional.

\subsubsection{El Instituto Antártico Chileno}

Desde el Año Geofísico Internacional (1957-1958), la actividad científica constituye uno de los principales actos de los países que tienen intereses antárticos. Chile, en su calidad de país antártico, que reclama soberanía sobre un sector de ese continente, ha desarrollado un activo y constante quehacer científico como contribución a esta acción y otras de carácter pacífico, según acuerdos internacionales que han logrado asegurar el éxito de estos propósitos.

El Instituto Antártico Chileno (INACH), fue creado mediante los artículos 5 y 6 de la Ley 15.266, que “Aprueba el Estatuto Orgánico del Ministerio de Relaciones Exteriores," del 10 de septiembre de 1963 y que fue publicado en el Diario Oficial del 10 de octubre de ese mismo año. La presentación oficial de la nueva entidad se produjo en un acto desarrollado el el 29 de mayo de 1964, en el Salón de Honor de la Universidad de Chile, misma casa de estudios que hoy nos acoje.

Número de página no utilizable para citar 
La principal misión del Instituto Antártico Chileno es planificar, coordinar, autorizar y realizar la actividad científica, tecnológica y de difusión en materias antárticas, rigiéndose por su estatuto orgánico. Para el cumplimiento de su misión, el instituto realiza y organiza todas las actividades operativas y logísticas que sean necesarias.

El año 2003 se producen cambios importantes en el Instituto Antártico Chileno, los que marcarán el inicio de una nueva etapa institucional. Como servicio público de carácter nacional, pasa a ejercer sus funciones en la ciudad de Punta Arenas. Esto representa una actividad concreta de descentralización y, al mismo tiempo, una decisión geopolítica de gran proyección para el país.

\subsubsection{La Política Antártica Nacional Chilena 2017}

La Política Antártica Nacional Chilena actualmente vigente fue promulgada por Decreto Supremo 56, del 24 de marzo de 2017, del Ministerio de Relaciones Exteriores.

La Política Antártica Nacional fija los objetivos de Chile en la Antártica. Ella es propuesta por el Consejo de Política Antártica y fijada por el Presidente de la República, quien la promulga mediante decreto supremo expedido por el Ministerio de Relaciones Exteriores, llevando las firmas de los Ministros de Defensa Nacional, Hacienda, Economía, Fomento y Turismo y Medio Ambiente.

Los objetivos de la Política Antártica Chilena son:

a) Proteger los derechos soberanos de Chile sobre el Territorio Chileno Antártico. Resguardar y promover los intereses nacionales en el Continente Antártico.

b) Consolidar una posición de liderazgo e influencia dentro del Sistema del Tratado Antártico, promoviendo asimismo el desarrollo de un régimen efectivo, asegurando la mantención de ese territorio libre de conflictos internacionales.

c) Proteger y promover el cuidado del medio ambiente antártico, incluyendo el medio ambiente marino y sus ecosistemas dependientes y asociados.

d) Desarrollar investigación en ciencias naturales y sociales sobre bases de excelencia, orientándose a temas globales de interés político y aquellos de interés económico nacional, a través de un Instituto Antártico Chileno robusto, eje de la colaboración científica internacional de Chile en la Antártica.

Número de página no utilizable para citar

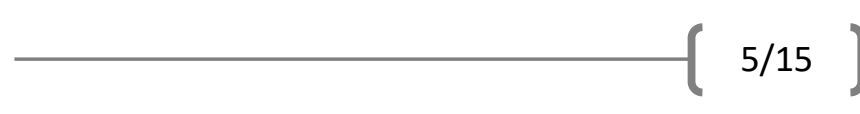


e) Desarrollar y promover a la Región de Magallanes y Antártica Chilena como un centro de actividad antártica nacional e internacional, incluyendo su consolidación como un polo de desarrollo científico y logístico para la Antártica. Reforzar la conectividad entre esta región y el continente antártico.

f) Facilitar el desarrollo de actividades económicas permitidas por el Sistema del Tratado Antártico, incluyendo la pesca sostenible y responsable, así como el turismo controlado y sustentable.

g) Fortalecer la conciencia e identidad antártica nacional, proteger y difundir el patrimonio histórico y cultural antártico de Chile y,

h) Perfeccionar la institucionalidad y la legislación antártica nacional con miras a facilitar la implementación de esta Política Antártica Nacional.

La Política Antártica Nacional debe ser sometida a evaluación y actualizada, al menos, cada 10 años desde la fecha de su promulgación.

\subsubsection{Plan Estratégico Antártico 2015-2019}

En la 51ºsesión del Consejo de Política Antártica (CPA), fue aprobado el Plan Estratégico 20152019, que organiza el quehacer antártico de las instituciones nacionales, y que contempla cinco áreas de trabajo: Fortalecer la institucionalidad antártica nacional; potenciar a la Región de Magallanes y la Antártica Chilena como puerta de entrada a la Antártica; promover la ciencia del país en el Sexto Continente y aumentar las capacidades operacionales y logísticas de Chile.

Los planes estratégicos antárticos tienen una vigencia de cuatro años, sin perjuicio que puedan ser modificados en un plazo menor si se estima necesario, y comprenderán las tareas y acciones concretas que se materializarán mediante el Programa Antártico Nacional de cada año.

El Ministerio de Relaciones Exteriores los debe desarrollar en base a los objetivos que establezca la Política Antártica Nacional, siendo éstos necesarios para orientar la acción de los ministerios y entidades con competencias sectoriales en la materia.

Para su elaboración, el Ministerio de Relaciones Exteriores se coordinará con los distintos ministerios y entidades con competencia antártica.

Número de página no utilizable para citar

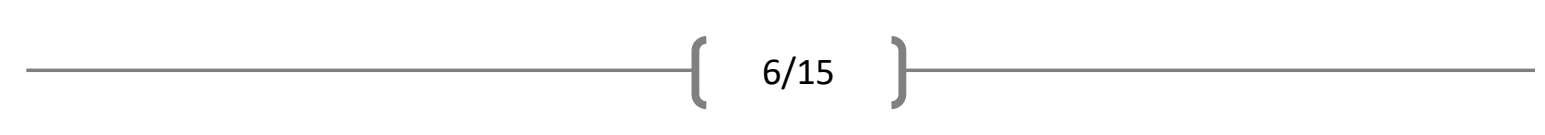




\subsubsection{El Programa Antártico Nacional}

El Programa Antártico Nacional es el conjunto de tareas y actividades concretas que se planifican anualmente para las campañas antárticas en cumplimiento del Plan Estratégico Antártico en vigor y de los objetivos de la Política Antártica Nacional, que coordina el Ministerio de Relaciones Exteriores, y está constituido por las siguientes actividades:

a) Actividades definidas por el Ministerio de Relaciones Exteriores para el cumplimiento de los objetivos de la Política Antártica Nacional, en atención a lo dispuesto en los Planes Estratégicos Antárticos;

b) Actividades de los operadores antárticos dependientes del Ministerio de Defensa Nacional en cuanto a la operación de sus bases y la logística propia, lo que informarán por intermedio del Ministerio de Defensa Nacional;

c) Actividades científicas y tecnológicas de investigación antártica en todas sus disciplinas, tanto ciencias naturales como ciencias sociales, jurídicas e históricas, coordinadas por el Instituto Antártico Chileno; y,

d) Cualquier otra actividad antártica nacional a cargo de entidades del Estado de Chile.

\subsection{Ministerio de Defensa Nacional}

\subsubsection{Subsecretaria de Defensa}

La Subsecretaría de Defensa, en virtud de lo dispuesto en los artículos 14 y 15 de la Ley 20.424 del 2010, que estable el Estatuto Orgánico del Ministerio de Defensa Nacional, es el órgano colaborador del Ministro de Defensa Nacional en asuntos de Política de Defensa, debiendo, entre otras tareas, coordinar la ejecución de aquella con la Política Antártica Nacional.

De esta manera, desde el 2010 a la fecha, esta subsecretaría ha cumplido un rol esencial al vincularse directamente, por una parte, con la Dirección Antártica de la Cancillería, y por otra con los operadores antárticos que dependen de cada una de las Fuerzas Armadas. Uno de los logros más claros de esta mayor y mejor coordinación, a la que se sumó además el Estado Mayor Conjunto, que es otro ente asesor del Ministerio de Defensa Nacional, ha sido la planificación y posterior operación de la Estación Polar Científica Conjunta Glaciar Unión. Ubicada, como su nombre lo indica, en el Glaciar Unión, en las cercanías del monte Ellsworth, esta base antártica de verano a solo 800 kilómetros del Polo Sur es la primera iniciativa realmente conjunta entre

Número de página no utilizable para citar

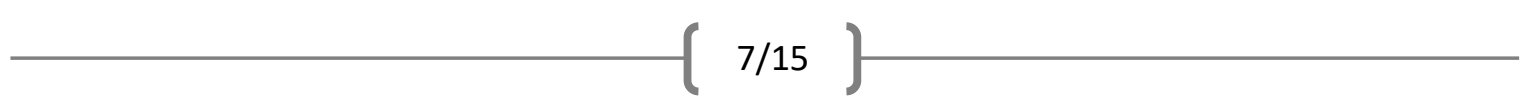


las tres Fuerzas Armadas y el Instituto Antártico de Chile, contando con un despliegue operativo y logístico, y un programa científico, de primer nivel. Solo tres países en el mundo, y Chile es uno de ellos, están en condiciones de operar al sur de los $80^{\circ}$ de latitud sur. Esta estación fue inaugurada el 4 de enero del 2014 por el Presidente Sebastián Piñera Echeñique.

\subsubsection{Operadores Antárticos Nacionales}

Los principales operadores antárticos nacionales son las unidades antárticas de las Fuerzas Armadas y el INACH. Las primeras son las encargadas de prestar el soporte operativo y logístico para todas las actividades antárticas chilenas, así como de mantener la presencia permanente de nuestro país, durante todo el año, en el Sexto Continente. Por su parte, y en estrecho vínculo con ellas, el INACH coordina y organiza la realización de las campañas científicas.

Estos operadores antárticos del Estado de Chile deben planificar y organizar su labor en coherencia con el Programa Antártico Nacional y en conformidad a los lineamientos que establezca el Consejo de Política Antártica.

En todas aquellas labores que corresponda que participen el personal o medios de más de una de las Fuerzas Armadas, asume también un rol preponderante en su coordinación y conducción el Estado Mayor Conjunto.

\subsection{Ministerio de Medioambiente. El Comité Operativo para la Evaluación de Impacto Ambiental sobre el Medioambiente Antártico}

El Comité Operativo para la Evaluación de Impacto Ambiental sobre el Medioambiente Antártico es el órgano encargado de evaluar en forma previa el impacto que pudieran producir en el medioambiente las actividades y proyectos que se planifique desarrollar en la Antártica. Para tal efecto, controla y certifica que cumplan con los requisitos ambientales dispuestos por las normas nacionales e internacionales respectivas.

El comité depende administrativamente del Ministerio de Medioambiente y su composición y normas de funcionamiento están determinadas por el reglamento respectivo, dictado por dicho ministerio, y que lleva además la firma del Ministro de Relaciones Exteriores.

Número de página no utilizable para citar

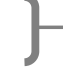




\subsection{Ministerio de Hacienda}

El Ministerio de Hacienda tiene como tarea dirigir la administración financiera del Estado.

En tal virtud, debe proponer la política económica y financiera del Gobierno en materias de su competencia y efectuar la coordinación y supervisión de las acciones que en virtud de ella se ejecuten.

Entre sus tareas está el elaborar el proyecto de presupuesto del Sector Público, así como el dictar las normas para su ejecución, administrando para tal efecto los recursos financieros del Estado, entre los cuales deben estar los fondos para financiar las actividades antárticas nacionales de los Operadores Antárticos.

\section{Reuniones y foros antárticos internacionales}

\subsection{El Sistema del Tratado Antártico}

Dentro del marco normativo establecido por el Sistema del Tratado Antártico, que como tal se compone de Tratado Antártico (1959, en vigor 1961), de la Convención para la Conservación de las Focas Antárticas (1972, en vigor 1978), de la Convención Conservación de los Recursos Vivos Marinos Antárticos (1980, en vigor 1982) y del Protocolo al Tratado Antártico sobre Protección del Medioambiente (1991, en vigor 1998), además de las múltiples regulaciones adoptadas en las Reuniones Consultivas del Tratado Antártica y las reuniones de la Comisión

para la Conservación de los Recursos Vivos Marinos Antárticos, se han ido desarrollando una serie de entidades e instituciones a través de las cuales los Estados llevan adelante el co-gobierno del Sexto Continente.

Analizaremos someramente las principales de ellas.

\subsection{Las Reuniones Consultivas del Tratado Antártico}

De 1961 a 1994 las Reuniones Consultivas se desarrollaron en general cada dos años, pero desde 1994 ellas se han celebrado anualmente. Los países anfitriones de estos encuentos son las Partes Consultivas, siguiendo el orden alfabético en inglés.

Número de página no utilizable para citar 
A la reunión asisten representantes de:

- $\quad$ Las Partes Consultivas;

- $\quad$ Partes no Consultivas;

- Observadores, que actualmente son el Comité Científico de Investigación Antártica (SCAR), la Comisión para la Conservación de los Recursos Vivos Marinos Antárticos (CCRVMA) y el Consejo de Administradores de los Programas Antárticos Nacionales (COMNAP); y

- Expertos invitados tales como la Coalición Antártica y del Océano Austral (ASOC) y la Asociación Internacional de Operadores Turísticos en la Antártida (IAATO).

Las Recomendaciones adoptadas entre 1961 y 1994, y desde entonces las Medidas, Decisiones y Resoluciones, son aprobadas en las Reuniones Consultivas por consenso. A través de ellas, se ponen en práctica los principios del Tratado Antártico y del Protocolo sobre Protección del Medioambiente, y proporcionandosé reglas y directrices para la gestión del área del Tratado Antártico y para el trabajo de las propias Reuniones Consultivas. Las Recomedaciones y las Medidas, cumplidos los procedimientos de rigor, constituyen normas obligatorias internacionalmente vinculantes para los Estados Consultivos. Las Decisiones también son obligatorias, pero en un ámbito mucho más acotado, ya que ellas solo disponen sobre asuntos organizativos internos de las Reuniones Consultivas. Por último, las Resoluciones son textos exhortatorios, que no son jurídicamente vinculantes para las Partes, aunque ciertamente consagran la opinión general sobre determinadas materias.

Sólo las Partes Consultivas participan en los procesos de la adopción de las decisiones. Sin embargo, los demás participantes en la reunión pueden contribuir a las deliberaciones.

\subsection{La Comisión para la Conservación de los Recursos Vivos Marinos Antárticos}

La participación de Chile en este foro internacional requiere de una coordinación estrecha de varios actores nacionales, tales como el Ministerio de Relaciones Exteriores a través de la Dirección de Antártica, el Ministerio de Medio Ambiente, la Subsecretaría de Pesca, el Servicio Nacional de Pesca, el Instituto de Fomento Pesquero, el Comité Oceanográfico Nacional, la Dirección General del Territorio Marítimo y Marina Mercante y el INACH.

Todos ellos actúan de manera coordinada a través de la Sección Nacional de la Comisión la que tiene su secretaria ejecutiva en la Dirección de Antártica del Ministerio de Relaciones Exteriores.

Número de página no utilizable para citar 
A través de la Sección Nacional y sus comités Científico y de Cumplimiento se verifica la implementación de las medidas de conservación y se propone la posición nacional para los distintos temas que son discutidos en la Comisión y otros comités de la Convención para la Conservación de los Recursos Vivos Marinos Antárticos. Asimismo, participan en ese diálogo permanente en calidad de invitados los representantes de la industria nacional con presencia en el área de la Convención.

\subsection{Consejo de Administradores de Programas Antárticos Nacionales}

El Consejo de Administradores de Programas Antárticos Nacionales (COMNAP) fue creado en 1988. Es la asociación internacional que reúne a todos los programas nacionales antárticos del mundo, con el objetivo de promocionar las mejores prácticas para apoyar la investigación científica en la Antártica.

Cada signatario del Tratado Antártico normalmente establece un Programa Nacional para trabajar en el Sexto Continente, el cual se efectúa bajo su responsabilidad nacional a fin de apoyar la realización de investigaciones científicas.

En la actualidad, en el COMNAP convergen los programas antárticos de 30 países de América, África, Asia, Europa y Oceanía.

\subsection{Reunión de Administradores de Programas Antárticos Latinoamericanos}

Anualmente, Argentina, Brasil, Chile, Uruguay, Ecuador y Perú participan en la Reunión de Administradores de Programas Antárticos Latinoamericanos (RAPAL), que constituye el foro de coordinación a nivel latinoamericano de temas de orden científico, logístico y ambiental que tienen relevancia en el área antártica. Las referidas Reuniones Latinoamericanas tuvieron su origen en las reuniones de los Directores de los Institutos Antárticos de Argentina, Chile y Uruguay que se realizaron en Buenos Aires (1987), Santiago (1988) y Montevideo (1989), respectivamente. A partir de 1990 a estas reuniones se incorporaron los operadores de los Programas Antárticos de Brasil, Perú y Ecuador.

Número de página no utilizable para citar 


\subsection{Comité Científico para la Investigación Antártica}

El Comité Científico para la Investigación Antártica (Scientific Committee on Antarctic Research, SCAR) es un comité del Consejo Internacional para la Ciencia. A la fecha, el SCAR tiene 44 Estados Miembros (32 en forma plena y 12 como Miembros Asociados), además de estar integrado por nueve asociaciones científicas.

El SCAR fue establecido luego de la reunión de Estocolmo del Consejo Internacional para la Ciencia, desarrollada entre el 9 y el 11 de septiembre de 1957. El primer encuentro del SCAR fue realizado en La Haya entre el 3 y 6 de febrero de 1958. Son países miembros plenos originales del mismo los siguientes 12 países: Argentina, Australia, Bélgica, Chile, Francia, Japón, Nueva Zelanda, Noruega, Unión Soviética, Sudáfrica, Reino Unido y Estados Unidos. Esto es, los mismos Estados que un año más tarde, en 1959, acodarían el Tratado Antártico.

El Comité se encarga de iniciar, desarrollar y coordinar la investigación científica en la Antártica. Asimismo, proporciona consejo científico a las Reuniones Consultivas del Tratado Antártico y de otras organizaciones en temas relacionados con la ciencia y con la conservación que afecta a la Antártica y al Océano Austral. El SCAR ha realizado numerosas recomendaciones que se han incorporado eventualemente como nomas dentro del Sistema del Tratado Antártico.

El SCAR se reúne cada dos años en un encuentro de delegados. Un comité ejecutivo escogido

por los delgados es el responsable de la administración día a día del Comité. La secretaría del SCAR se encuentra en el Scott Polar Research Institute, en Cambridge, Reino Unido.

El SCAR celebra, con anterioridad a la elección de los delegados, una conferencia científica abierta, encaminada a llamar la atención en temas antárticos así como reuniones de los grupos científicos que diseñan los programas científicos que serán, posteriormente, aprobados por los delegados.

Los Profesores Jabour, Shibata, Ferrada y Ramírez, aquí presentes, integran el Comité Permanente de Humanidades y Ciencias Sociales del SCAR. 


\section{Diálogos bilaterales y multilaterales en el ámbito antártico}

En materia de diálogo antártico, el Ministerio de Relaciones Exteriores, a través de la Dirección de Antártica, mantiene un constante intercambio y relacionamiento bilateral y multilateral con múltiples países.

En lo bilateral se celebran semestral o anualmente, reuniones con países tales como Argentina, Estados Unidos de Norteamérica, el Reino Unido de Gran Bretaña e Irlanda del Norte, Noruega y Corea del Sur.

En lo multilateral se celebran reuniones con los países reclamantes de territorio antártico, a saber, Argentina, Francia, Reino Unido de Gran Bretaña e Irlanda del Norte, Noruega, Nueva Zelandia y Australia.

Asimismo, se mantiene un permanente diálogo con los países que conforman el P5 (Miembros Permanentes del Consejo de Seguridad de Naciones Unidas), incorporándose China a los ya citados anteriormente.

\section{Una nueva Ley Antártica para Chile}

Antes de concluir, resulta de interés hacer algunas breves reflexiones sobre una de las iniciativas jurídicas antártica de mayor importancia actual: la elaboración de un nuevo Estatuto Antártico para Chile. El vigente, data de 1956.

En materia de regulación antártica Chile cuenta con dos grandes desafíos. Por una parte, desde el siglo XIX a la fecha han dictado cerca de 300 normas legales y reglamentarias que de un modo relativamente directo se refieren a la Antártica. Muchas de ellas están formalmente en vigor. Por otra, Chile ha asumido una serie de compromisos internacionales en la materia, para cuyo cumplimiento requiere contar con un marco normativo adecuado. Por ello, la necesidad de sistematizar, armonizar y modernizar la normativa antártica chilena aparece como una tarea prioritaria, a fin de potenciar los esfuerzos por conservar y afianzar nuestros derechos soberanos y al mismo tiempo dar cumplimiento a los compromisos internacionales.

Número de página no utilizable para citar 
La primera instrucción formal de iniciar los trabajos respectivos se dio el año 2007, en una reunión del Consejo de Política Antártica y a iniciativa del embajador Jorge Berguño, uno de nuestros grandes prohombres antárticos, juntos a Oscar Pinochet de la Barra y varios más. Sin embargo, no existieron avances sustantivos sino hasta el año 2011, cuando se crea la Dirección de Antártica de la Cancillería, que tuve el honor de dirigir en esa primera fase. Junto a la subdirectora, y alumna de esta Escuela, María Luisa Carvallo, y al organizador de estas jornadas, profesor Luis Valentín Ferrada, emprendimos un trabajo titánico, durante más de dos años en que nos reuníamos una o dos veces a la semana, hasta que logramos completar el primer borrador del proyecto de ley completo, en abril del 2013.

Como suele ocurrir en estos procesos pre-legislativos y legislativos, los tiempos y los textos avanzan a veces de modo azaroso y en otros se detienen por meses o años. En definitiva, el proyecto de ley fue ingresado a la Cámara de Diputados en marzo del 2014, sin tener mayor movimiento hasta marzo del 2017, pero retomando su tramitación progresiva solo a mediados del año pasado. Se le ingresaron un conjunto de indicaciones en octubre del 2018 y, en definitiva, la Comisión de Zonas Extremas y Antártica Chilena de la Cámara de Diputados lo aprobó en el mes de diciembre, y la Sala lo hizo en enero de este año. Actualmente se encuentra en segundo trámite constitucional en el Senado, y trabajamos en un nuevo conjunto de indicaciones.

Nos interesa tener una nueva ley antártica, pero, más que eso, nos interesa tener una muy buena ley, que de cuenta de los desafíos de hoy y del futuro.

\section{Conclusiones}

Como se ha visto, la institucionalidad antártica, se compone de un conjunto coordinado de entidades nacionales e internacionales, con distintas competencias, pero con objetivos que en uno y otro ámbito tienden en sentidos equivalentes.

Uno de los factores más característicos de todo lo antártico, pero que desgraciadamente no siempre se entiende, es la doble dimensión nacional / internacional de todo cuanto allí se hace. Chile defiende sus derechos soberanos, pero lo hace en el marco del Sistema del Tratado Antártico. Esta naturaleza dual obliga a actuar siempre con prudencia y cuidado, comprendido las consecuencias internacionales que tiene todo cuanto allí se haga, pero también asumiendo que esa acción exterior redunda en la protección de nuestros derechos. Todo esto hace que la institucionalidad antártica esté ligada de un modo tan directo con las competencias del Ministerio

Número de página no utilizable para citar 
Revista Tribuna Internacional

Volumen $8 \cdot \mathrm{N}^{\mathrm{o}} 15 \cdot 2019$

ISSN 0719-482X (versión en línea)

de Relaciones Exteriores, y que sea esta cartera la que está en una mejor posición para ejercer la coordinación de la actividad nacional en la Antártica y propender a la materialización de la Política Antártica, correspondiéndole colaborar con el Presidente de la República en la planificación, conducción, coordinación, ejecución, control e información de todo cuanto Chile haga en relación con el Continente Antártico. 